\title{
End-point estimates and multi-parameter paraproducts on higher dimensional tori
}

\section{John T. Workman}

\begin{abstract}
Analogues of multi-parameter multiplier operators on $\mathbb{R}^{d}$ are defined on the torus $\mathbb{T}^{d}$. It is shown that these operators satisfy the classical Coifman-Meyer theorem. In addition, $L(\log L)^{n}$ end-point estimates are proved
\end{abstract}

\section{Introduction}

This article is, in part, a continuation of $[13,14]$. It is also derived from the author's dissertation, which can be found in full at [17].

Recall the multi-linear Coifman-Meyer [5] operator

$$
\Lambda_{m}^{(1)}\left(f_{1}, \ldots, f_{d}\right)(x)=\int_{\mathbb{R}^{d}} m(t) \widehat{f}_{1}\left(t_{1}\right) \cdots \widehat{f}_{d}\left(t_{d}\right) e^{2 \pi i x\left(t_{1}+\cdots+t_{d}\right)} d t,
$$

for Schwartz functions $f_{j}$ and where $m$ satisfies a standard MarcinkiewiczMihlin-Hörmander type condition [12]. It is well known this operator maps $L^{p_{1}} \times \cdots \times L^{p_{d}} \rightarrow L^{p}$ for $1 / p_{1}+\cdots+1 / p_{d}=1 / p$ and $1<p_{j}<\infty$. The case when $p \geq 1$ was originally shown by Coifman and Meyer. The general case $p>1 / d$ was settled later in $[9,11]$.

Led by natural questions in non-linear partial differential equations, extensions of this operator were considered by Muscalu et. al.: first the socalled bi-parameter multiplier [13], then multi-parameter multipliers [14]. In this setting, $m$ is allowed to belong to a much wider class of multipliers which behave like the product of standard multipliers. Special cases of these multiplier operators had been previously considered by Christ and Journé $[4,10]$. In $[13,14]$, it is shown that these multiplier operators satisfy the same $L^{p_{1}} \times \cdots \times L^{p_{d}} \rightarrow L^{p}$ property.

2000 Mathematics Subject Classification: 42B15.

Keywords: Paraproduct, polydisc. 
However, in the single-parameter case of Coifman and Meyer, more is known. We have "end-point" estimates corresponding to the case when any or all of the $p_{j}$ are equal to 1 . Here, the result is $L^{p_{1}} \times \cdots \times L^{p_{d}} \rightarrow L^{p, \infty}$. In the multi-parameter setting, no such end-point estimates are known.

A natural candidate for such an estimate would involve $L \log L$ spaces, because of how they arise in interpolation results. Naively, an operator which maps $L^{1} \rightarrow L^{1, \infty}$, and also satisfies some $L^{p}$ result, is often thought to also satisfy some $L \log L$ to $L^{1}$ property. Indeed, we recall the result of Stein [16], which states $M f$ is locally integrable if and only if $f$ is locally in $L \log L$; alternatively, C. Fefferman [6] showed the maximal double Hilbert transform maps $L \log L\left([0,1]^{2}\right)$ to $L^{1, \infty}\left([0,1]^{2}\right)$.

That $L \log L$ estimates can only be gained in the compact setting is a rather common obstacle. To avoid this, we instead consider analogues of multiplier operators defined on the torus $\mathbb{T}^{d}$. This also allows a departure from the classical definition of $L \log L$ spaces to a more iterative approach which blends perfectly with our methods. Ultimately, we show that the $s$-parameter multiplier operator $\Lambda_{m}^{(s)}$ in this setting satisfies the classical Coifman-Meyer theorem, along with the desired end-point estimate: for $p_{j}=1$ each $L^{p_{j}}$ is replaced by $L(\log L)^{s-1}$.

The organization is as follows. In the next section, characterizations of $L(\log L)^{n}$ are developed for any probability space, and several important results therein are proved. Section 3 details the connections between $L(\log L)^{n}$ spaces and the Hardy-Littlewood maximal operator. Section 4 deals with the notion of adapted families and a particular square function of Littlewood-Paley type. Section 5 introduces hybrid square-max operators. In Section 6, bi-parameter multiplier operators are handled, while section 7 is a non-rigorous survey of the proof for multi-parameter multipliers.

A remark on the notation used: we will write $A \lesssim B$ whenever $A \leq C \cdot B$ with some universal constant $C$.

\section{Zygmund spaces and $L(\log L)^{n}$}

Let $(X, \rho)$ be a probability space. For $f:(X, \rho) \rightarrow \mathbb{C}$, denote the decreasing rearrangement of $f$ by $f^{*}$.

Definition. For $t>0$ and $f:(X, \rho) \rightarrow \mathbb{C}$, let $f^{(*, 1)}(t)=f^{*}(t)$ and for integers $n \geq 2$, set $f^{(*, n)}(t)=\frac{1}{t} \int_{0}^{t} f^{(*, n-1)}(s) d s$.

On a probability space, $f^{*}$ is supported on $[0,1]$. It is advantageous to informally think of each $f^{(*, n)}$ as being defined only on $(0,1]$.

We can immediately verify the following properties: (1) $f^{(*, n)}$ is nonnegative, decreasing, and identically 0 if and only if $f=0$ a.e. $[\rho] ;(2) f^{(*, n)} \leq$ 
$f^{(*, n+1)} ;(3)(\alpha f)^{(*, n)}=|\alpha| f^{(*, n)}(4)|f| \leq|g|$ a.e. $[\rho]$ implies $f^{(*, n)} \leq g^{(*, n)}$ pointwise; (5) $\left|f_{k}\right| \uparrow|f|$ a.e. $[\rho]$ implies $f_{k}^{(*, n)} \uparrow f^{(*, n)}$ pointwise.

We would also like to show $(f+g)^{(*, n)}(t) \leq f^{(*, n)}(t)+g^{(*, n)}(t)$ for all $t>0$ and $n \geq 2$; this property does not hold for $n=1$. By induction, it suffices to prove the result for $n=2$. However, this is an immediate consequence of the following technical result of Bennett and Sharpley [3]:

$$
t f^{(*, 2)}(t)=\int_{0}^{t} f^{*}(s) d s=\inf _{f=g+h}\left\{\|g\|_{1}+t\|h\|_{\infty}\right\} .
$$

Definition. For $f:(X, \rho) \rightarrow \mathbb{C}$ and integers $n \geq 0$, define $\|f\|_{L(\log L)^{n}}$ by

$$
\|f\|_{L(\log L)^{n}}=\int_{0}^{1} f^{(*, n+1)}(t) d t .
$$

Define the Zygmund space $L(\log L)^{n}(X)$ as the set of functions $f$ with $\|f\|_{L(\log L)^{n}}<\infty$.

We note that $L(\log L)^{0}(X)=L^{1}(X)$, which is a useful notational shortcut. Clearly, $\|\cdot\|_{L(\log L)^{n}}$ is a norm with the additional properties that $|f| \leq|g|$ a.e. $[\rho]$ implies $\|f\|_{L(\log L)^{n}} \leq\|g\|_{L(\log L)^{n}}$ and $\left|f_{k}\right| \uparrow|f|$ a.e. $[\rho]$ implies $\left\|f_{k}\right\|_{L(\log L)^{n}} \uparrow\|f\|_{L(\log L)^{n}}$. Further, this definition of $L(\log L)^{n}$ coincides with the classical space.

Theorem 2.1. $f \in L(\log L)^{n}(X)$ if and only if

$$
\int_{X}|f(x)|\left(\log ^{+}|f(x)|\right)^{n} \rho(d x)<\infty .
$$

The proof is fairly technical but straightforward and is left to the reader. Using Hardy's inequality, it is also easy to establish the following.

Theorem 2.2. For any $1<p \leq \infty$ and $n \geq 0$,

$$
L^{p}(X) \subseteq L(\log L)^{n+1}(X) \subseteq L(\log L)^{n}(X) \subseteq L^{1}(X),
$$

with $\|f\|_{1} \leq\|f\|_{L(\log L)^{n}} \leq\|f\|_{L(\log L)^{n+1}} \lesssim\|f\|_{p}$.

The principal reason for defining $L(\log L)^{n}$ as we have is the ease in which we gain interpolation results.

Lemma 2.3. Let $T$ be a sublinear operator which maps $L^{1}(X) \rightarrow L^{1, \infty}(X)$ and $L^{p}(X) \rightarrow L^{q, \infty}(X)$, for some $1<p, q<\infty$. Then, for $n \in \mathbb{N}$,

$$
(T f)^{(*, n)}(t) \lesssim\left[\frac{1}{t} \int_{0}^{t^{m}} f^{(*, n)}(s) d s+t^{-1 / q} \int_{t^{m}}^{1} s^{1 / p-1} f^{(*, n)}(s) d s\right],
$$

where $m=\left(\frac{1}{q}-1\right)\left(\frac{1}{p}-1\right)^{-1}$. 
Proof. We show this by induction. The $n=1$ case is a technical result established in [3]. Assume it is true for $n-1$. Then,

$$
\begin{aligned}
& (T f)^{(*, n)}(t)=\frac{1}{t} \int_{0}^{t} T^{(*, n-1)}(s) d s \\
& \quad \lesssim \frac{1}{t} \int_{0}^{t} \frac{1}{s} \int_{0}^{s^{m}} f^{(*, n-1)}(u) d u d s+\frac{1}{t} \int_{0}^{t} s^{-1 / q} \int_{s^{m}}^{1} u^{1 / p-1} f^{(*, n-1)}(u) d u d s \\
& \quad=: I+I I .
\end{aligned}
$$

By the change of variables $r=s^{m}$,

$$
I=\frac{1}{m} \frac{1}{t} \int_{0}^{t^{m}} \frac{1}{r} \int_{0}^{r} f^{(*, n-1)}(u) d u d r=\frac{1}{m} \frac{1}{t} \int_{0}^{t^{m}} f^{(*, n)}(r) d r .
$$

On the other hand, changing the order of integration gives

$$
\begin{aligned}
I I= & \frac{1}{t} \int_{0}^{t^{m}} u^{1 / p-1} f^{(*, n-1)}(u) \int_{0}^{u^{1 / m}} s^{-1 / q} d s d u \\
& +\frac{1}{t} \int_{t^{m}}^{1} u^{1 / p-1} f^{(*, n-1)}(u) \int_{0}^{t} s^{-1 / q} d s d u \\
= & \frac{1}{1-1 / q} \frac{1}{t} \int_{0}^{t^{m}} f^{(*, n-1)}(u) d u+\frac{1}{1-1 / q} t^{-1 / q} \int_{t^{m}}^{1} u^{1 / p-1} f^{(*, n-1)}(u) d u \\
\leq & \frac{1}{1-1 / q}\left[\frac{1}{t} \int_{0}^{t^{m}} f^{(*, n)}(u) d u+t^{-1 / q} \int_{t^{m}}^{1} u^{1 / p-1} f^{(*, n)}(u) d u\right] .
\end{aligned}
$$

Theorem 2.4. Let $T$ be a sublinear operator which maps $L^{1}(X) \rightarrow L^{1, \infty}(X)$ and $L^{p}(X) \rightarrow L^{q, \infty}(X)$, for some $1<p, q<\infty$. Then, for all $n \in \mathbb{N}, T$ also maps $L(\log L)^{n}(X) \rightarrow L(\log L)^{n-1}(X)$.

Proof. Set $m=\left(\frac{1}{q}-1\right)\left(\frac{1}{p}-1\right)^{-1}$. Using Lemma 2.3 and the same change of variables and Fubini arguments,

$$
\begin{aligned}
\|T f\|_{L(\log L)^{n-1}}=\int_{0}^{1}(T f)^{(*, n)}(t) d t \\
\quad \lesssim \int_{0}^{1} \frac{1}{t} \int_{0}^{t^{m}} f^{(*, n)}(s) d s d t+\int_{0}^{1} t^{-1 / q} \int_{t^{m}}^{1} s^{1 / p-1} f^{(*, n)}(s) d s d t \\
\quad=\frac{1}{m} \int_{0}^{1} \frac{1}{u} \int_{0}^{u} f^{(*, n)}(s) d s d u+\int_{0}^{1} s^{1 / p-1} f^{(*, n)}(s) \int_{0}^{s^{1 / m}} t^{-1 / q} d t d s \\
\quad=\frac{1}{m} \int_{0}^{1} f^{(*, n+1)}(u) d u+\frac{1}{1-1 / q} \int_{0}^{1} f^{(*, n)}(s) d s \lesssim\|f\|_{L(\log L)^{n}} .
\end{aligned}
$$


Corollary 2.5. Let $T$ be a sublinear operator. If for some $1<p, r<\infty$

$$
\begin{gathered}
\left\|\left(\sum_{k=1}^{\infty}\left|T f_{k}\right|^{r}\right)^{1 / r}\right\|_{1, \infty} \lesssim\left\|\left(\sum_{k=1}^{\infty}\left|f_{k}\right|^{r}\right)^{1 / r}\right\|_{1} \text { and } \\
\left\|\left(\sum_{k=1}^{\infty}\left|T f_{k}\right|^{r}\right)^{1 / r}\right\|_{p} \lesssim\left\|\left(\sum_{k=1}^{\infty}\left|f_{k}\right|^{r}\right)^{1 / r}\right\|_{p}
\end{gathered}
$$

then for all $n \in \mathbb{N}$

$$
\left\|\left(\sum_{k=1}^{\infty}\left|T f_{k}\right|^{r}\right)^{1 / r}\right\|_{L(\log L)^{n-1}} \lesssim\left\|\left(\sum_{k=1}^{\infty}\left|f_{k}\right|^{r}\right)^{1 / r}\right\|_{L(\log L)^{n}} .
$$

Proof. This only requires viewing the above theory through the wider scope of Banach space-valued functions $f:(X, \rho) \rightarrow\left(B,\|\cdot\|_{B}\right)$ (see [8]). If instead one defined the decreasing rearrangement $f^{*}$ for Banach space-valued functions, in the natural way, and repeated the definitions and arguments of this section, everything would still hold. In particular, the previous theorem is valid; if $T$ is sublinear operator mapping $L_{B}^{1}(X)$ to $L_{B}^{1, \infty}(X)$ and $L_{B}^{p}(X)$ to $L_{B}^{q, \infty}(X)$, then $T: L(\log L)_{B}^{n}(X) \rightarrow L(\log L)_{B}^{n-1}(X)$. But, simply by definition, $f^{*}(t)=\left(\|f\|_{B}\right)^{*}(t)$, where $\left(\|f\|_{B}\right)^{*}$ is understood as the decreasing rearrangement of the map $x \mapsto\|f(x)\|_{B}$. Thus,

$$
\|f\|_{L(\log L)_{B}^{n}}=\|\| f\left\|_{B}\right\|_{L(\log L)^{n}} .
$$

Let $B=\ell^{r}$ and $\bar{T}(f)=\left(T f_{1}, T f_{2}, \ldots\right)$, so that $\bar{T}: L_{B}^{1}(X) \rightarrow L_{B}^{1, \infty}(X)$ and $L_{B}^{p}(X) \rightarrow L_{B}^{p}(X)$. Thus, $\bar{T}: L(\log L)_{B}^{n}(X) \rightarrow L(\log L)_{B}^{n-1}(X)$, which is what was promised.

\section{Connections to Hardy-Littlewood}

Let us turn our attention to the probability space $(\mathbb{T}, m)$. Let $M f$ denote the standard Hardy-Littlewood maximal operator on $\mathbb{T}$. Of course, $M$ maps $L^{1}(\mathbb{T}) \rightarrow L^{1, \infty}(\mathbb{T})$ and $L^{p}(\mathbb{T}) \rightarrow L^{p}(\mathbb{T})$ for all $1<p<\infty$. So, by the interpolation results of the previous section, $M: L(\log L)^{n}(\mathbb{T}) \rightarrow L(\log L)^{n-1}(\mathbb{T})$. Further, from Fefferman and Stein [7], we know

$$
\begin{gathered}
\left\|\left(\sum_{k=1}^{\infty}\left|M f_{k}\right|^{r}\right)^{1 / r}\right\|_{1, \infty} \lesssim\left\|\left(\sum_{k=1}^{\infty}\left|f_{k}\right|^{r}\right)^{1 / r}\right\|_{1} \text { and } \\
\left\|\left(\sum_{k=1}^{\infty}\left|M f_{k}\right|^{r}\right)^{1 / r}\right\|_{p} \lesssim\left\|\left(\sum_{k=1}^{\infty}\left|f_{k}\right|^{r}\right)^{1 / r}\right\|_{p},
\end{gathered}
$$

for all $1<p, r<\infty$, and therefore Corollary 2.5 applies. However, much more can be said. 
Theorem 3.1. $f^{(*, n+1)}(t) \sim(M f)^{(*, n)}(t)$, where the underlying constants do not depend on $f$ or $t$.

It clearly suffices, by induction, to prove $f^{(*, 2)}(t) \sim(M f)^{*}(t)$. But, this is a well-known result; see [2, 3].

Corollary 3.2. $f \in L(\log L)^{n+1}(\mathbb{T})$ if and only if $M f \in L(\log L)^{n}(\mathbb{T})$, and, in particular, $\|f\|_{L(\log L)^{n+1}} \sim\|M f\|_{L(\log L)^{n}}$.

\section{Adapted families}

Definition. A smooth function $\varphi: \mathbb{T} \rightarrow \mathbb{C}$ is adapted to an interval $I$ with constants $C_{m}>0, m \in \mathbb{N}$, if

$$
\begin{aligned}
& |\varphi(x)| \leq C_{m}\left(1+\frac{\operatorname{dist}_{\mathbb{T}}(x, I)}{|I|}\right)^{-m} \text { for all } x \in \mathbb{T}, m \in \mathbb{N} \\
& \left|\varphi^{\prime}(x)\right| \leq C_{m} \frac{1}{|I|}\left(1+\frac{\operatorname{dist}_{\mathbb{T}}(x, I)}{|I|}\right)^{-m} \text { for all } x \in \mathbb{T}, m \in \mathbb{N}
\end{aligned}
$$

A family of smooth functions $\varphi_{I}: \mathbb{T} \rightarrow \mathbb{C}$, indexed by the dyadic intervals, is called an adapted family if each $\varphi_{I}$ is adapted to $I$ with the same universal constants. We say $\left\{\varphi_{I}\right\}_{I}$ is a 0 -mean adapted family if it is an adapted family, with the additional property that $\int_{\mathbb{T}} \varphi_{I} d m=0$ for all $I$.

For an adapted family $\varphi_{I}$, define $\phi_{I}=|I|^{-1 / 2} \varphi_{I}$, where $|I|$ denotes Lebesgue measure. Note $\left\|\phi_{I}\right\|_{2} \lesssim 1$ for all $I$. Often, $\phi_{I}$ is called an $L^{2}$ normalized family. Per our notation, $\varphi_{I}$ will always represent an adapted family, and $\phi_{I}$ will always represent the $L^{2}$-normalization.

Conceptually, we often think of functions which are adapted to an interval $I$ as being "almost supported" in $I$. The following theorem, which is a variation of a result in [14], gives some rigid meaning to this.

Theorem 4.1. Let $\varphi_{I}: \mathbb{T} \rightarrow \mathbb{C}$ be adapted to a dyadic interval $I$, with $|I|=2^{-N}$. Then, we can write

$$
\varphi_{I}=\sum_{k=1}^{\infty} 2^{-10 k} \varphi_{I}^{k},
$$

where each $\varphi_{I}^{k}$ is adapted to $I$, uniformly in $k$, with $\operatorname{supp}\left(\varphi_{I}^{k}\right) \subseteq 2^{k} I$ for $1 \leq k \leq N$ and $\varphi_{I}^{k}=0$ otherwise. Further, if $\varphi_{I}$ has integral 0 , each $\varphi_{I}^{k}$ can be chosen to have integral 0.

To clarify the notation above, for an interval $I$ and constant $\alpha>0, \alpha I$ is the interval concentric with $I$ so that $|\alpha I|=\alpha|I|$. 
Given an adapted family $\varphi_{I}$, its normalization $\phi_{I}$, and $f: \mathbb{T} \rightarrow \mathbb{C}$, we will be interested in "averages" of $f$ with respect to the family. Let

$$
M^{\prime} f(x)=\sup _{I} \frac{1}{|I|^{1 / 2}}\left|\left\langle\phi_{I}, f\right\rangle\right| \chi_{I}(x) \text {. }
$$

where the supremum is over all dyadic intervals. For a 0-mean adapted family $\varphi_{I}$, define the Littlewood-Paley (discrete) square function by

$$
S f(x)=\left(\sum_{I} \frac{\left|\left\langle\phi_{I}, f\right\rangle\right|^{2}}{|I|} \chi_{I}(x)\right)^{1 / 2},
$$

where the sum is over all dyadic intervals.

Using Theorem 4.1 , it is easily shown that $M^{\prime} f \lesssim M f$, so that $M^{\prime}$ satisfies the same properties as $M$. It is known that $S: L^{1} \rightarrow L^{1, \infty}$ and $L^{p} \rightarrow L^{p}$ for $1<p<\infty$ (see [17] for a new approach). We will need to establish Fefferman-Stein inequalities for $S$ as well, but the only the special case $r=2$ will be necessary.

Theorem 4.2. For $1<p<\infty$ and any sequence $f_{1}, f_{2}, \ldots$ of complex-valued functions on $\mathbb{T}$

$$
\begin{gathered}
\left\|\left(\sum_{k=1}^{\infty}\left|S f_{k}\right|^{2}\right)^{1 / 2}\right\|_{p} \lesssim\left\|\left(\sum_{k=1}^{\infty}\left|f_{k}\right|^{2}\right)^{1 / 2}\right\|_{p}, \\
\left\|\left(\sum_{k=1}^{\infty}\left|S f_{k}\right|^{2}\right)^{1 / 2}\right\|_{1, \infty} \lesssim\left\|\left(\sum_{k=1}^{\infty}\left|f_{k}\right|^{2}\right)^{1 / 2}\right\|_{1}
\end{gathered}
$$

Only considering the $r=2$ allows us to use Rademacher functions and Khinchine's inequality to "linearize." For the weak- $L^{1}$ inequality, an alternate characterization called the Kolmogorov condition is helpful (see [8]). For full details, see [17].

\section{Hybrid operators}

The definitions of the hybrid operators $M S, S M$, and $S S$, their properties, and their relevance in our context are borrowed from [13].

We say a set $R \subset \mathbb{T}^{2}$ is a dyadic rectangle if there exist dyadic intervals $I$ and $J$ so that $R=I \times J$. Given two (possibly distinct) adapted families $\varphi_{I}$ and $\varphi_{J}$, we will write $\varphi_{R}(x, y)=\varphi_{I}(x) \varphi_{J}(y)$. For $\varphi_{R}=\varphi_{I} \otimes \varphi_{J}$, set $\phi_{R}=|R|^{-1 / 2} \varphi_{R}=\phi_{I} \otimes \phi_{J}$. 
For functions $f: \mathbb{T}^{2} \rightarrow \mathbb{C}$, define

$$
M M f(x, y)=\sup _{R} \frac{1}{|R|^{1 / 2}}\left|\left\langle\phi_{R}, f\right\rangle\right| \chi_{R}(x, y) .
$$

If $\left\{\varphi_{R}\right\}$ is a family such that $\int_{\mathbb{T}} \varphi_{J} d m=0$ for all $J$, then define

$$
M S f(x, y)=\sup _{I} \frac{1}{|I|^{1 / 2}}\left(\sum_{J} \frac{\left|\left\langle\phi_{R}, f\right\rangle\right|^{2}}{|J|} \chi_{J}(y)\right)^{1 / 2} \chi_{I}(x),
$$

Analogously, if $\int_{\mathbb{T}} \varphi_{I} d m=0$ for all $I$, define

$$
S M f(x, y)=\left(\sum_{I} \frac{\left(\sup _{J} \frac{1}{|J|^{1 / 2}}\left|\left\langle\phi_{R}, f\right\rangle\right| \chi_{J}(y)\right)^{2}}{|I|} \chi_{I}(x)\right)^{1 / 2} .
$$

Finally, if $\int_{\mathbb{T}} \varphi_{I} d m=\int_{\mathbb{T}} \varphi_{J} d m=0$, set

$$
S S f(x, y)=\left(\sum_{R} \frac{\left|\left\langle\phi_{R}, f\right\rangle\right|^{2}}{|R|} \chi_{R}(x, y)\right)^{1 / 2} .
$$

Theorem 5.1. Each of $M M, M S, S M$, and $S S$ maps $L^{p}\left(\mathbb{T}^{2}\right) \rightarrow L^{p}\left(\mathbb{T}^{2}\right)$ for all $1<p<\infty, L(\log L)^{n+2}\left(\mathbb{T}^{2}\right) \rightarrow L(\log L)^{n}\left(\mathbb{T}^{2}\right)$ for all $n \geq 0$, and $L \log L\left(\mathbb{T}^{2}\right) \rightarrow L^{1, \infty}\left(\mathbb{T}^{2}\right)$.

Proof. Let $M_{S}$ denote the strong maximal operator (that is, where the supremum is taken over all bi-parameter rectangles). Define the $1^{\text {st }}$ and $2^{\text {nd }}$ variables maximal operators $M_{1}$ and $M_{2}$ as follows. For $f: \mathbb{T}^{2} \rightarrow \mathbb{C}$, let $M_{1} f\left(x_{1}, x_{2}\right)=M\left(f\left(\cdot, x_{2}\right)\right)\left(x_{1}\right)$ and $M_{2} f\left(x_{1}, x_{2}\right)=M\left(f\left(x_{1}, \cdot\right)\right)\left(x_{2}\right)$. It is clear that $M_{1}, M_{2}$ satisfy all the $L^{p}$ properties and Fefferman-Stein inequalities that $M$ does. Define $M_{1}^{\prime}, M_{2}^{\prime}, S_{1}, S_{2}$ similarly.

Using Theorem 4.1 as before, $M M f \lesssim M_{S} f$. But, $M_{S} f \leq M_{1} \circ M_{2} f$, so that

$$
\begin{aligned}
\|M M f\|_{p} & \lesssim\left\|M_{1} \circ M_{2} f\right\|_{p} \lesssim\left\|M_{2} f\right\|_{p} \lesssim\|f\|_{p} \\
\|M M f\|_{L(\log L)^{n}} & \lesssim\left\|M_{1} \circ M_{2} f\right\|_{L(\log L)^{n}} \lesssim\left\|M_{2} f\right\|_{L(\log L)^{n+1}} \lesssim\|f\|_{L(\log L)^{n+2}} \\
\|M M f\|_{1, \infty} & \lesssim\left\|M_{1} \circ M_{2} f\right\|_{1, \infty} \lesssim\left\|M_{2} f\right\|_{1} \lesssim\|f\|_{L \log L}
\end{aligned}
$$

We abuse notation slightly and write $\left\langle f, \phi_{I}\right\rangle$ to mean $\int_{\mathbb{T}} \bar{\phi}_{I}(x) f(x, y) d x$, a function of the variable $y$. Thus, $\left\langle\phi_{R}, f\right\rangle=\left\langle\phi_{J},\left\langle f, \phi_{I}\right\rangle\right\rangle$ makes sense. Also, 
we can consider the two variable function $\left\langle f, \phi_{I}\right\rangle \chi_{I}$. In this manner,

$$
\begin{aligned}
\operatorname{SMf}(x, y) & =\left(\sum_{I} \frac{\left(\sup _{J} \frac{1}{|J|^{1 / 2}}\left|\left\langle\phi_{R}, f\right\rangle\right| \chi_{J}(y)\right)^{2}}{|I|} \chi_{I}(x)\right)^{1 / 2} \\
& =\left(\sum_{I}\left(\sup _{J} \frac{1}{|J|^{1 / 2}}\left|\left\langle\phi_{J}, \frac{\left\langle f, \phi_{I}\right\rangle}{|I|^{1 / 2}} \chi_{I}(x)\right\rangle\right| \chi_{J}(y)\right)^{2}\right)^{1 / 2} \\
& =\left(\sum_{I} M_{2}^{\prime}\left(\frac{\left\langle f, \phi_{I}\right\rangle}{|I|^{1 / 2}} \chi_{I}\right)(x, y)^{2}\right)^{1 / 2} .
\end{aligned}
$$

By the Fefferman-Stein inequalities on $M^{\prime}$ ( or $M_{2}^{\prime}$ ),

$$
\begin{aligned}
\|S M f\|_{p} & =\left\|\left(\sum_{I} M_{2}^{\prime}\left(\frac{\left\langle f, \phi_{I}\right\rangle}{|I|^{1 / 2}} \chi_{I}\right)^{2}\right)^{1 / 2}\right\|_{p} \\
& \lesssim\left\|\left(\sum_{I} \frac{\left|\left\langle f, \phi_{I}\right\rangle\right|^{2}}{|I|} \chi_{I}\right)^{1 / 2}\right\|_{p}=\left\|S_{1} f\right\|_{p} \lesssim\|f\|_{p}
\end{aligned}
$$

and

$$
\begin{aligned}
\|S M f\|_{L(\log L)^{n}} & =\left\|\left(\sum_{I} M_{2}^{\prime}\left(\frac{\left\langle f, \phi_{I}\right\rangle}{|I|^{1 / 2}} \chi_{I}\right)^{2}\right)^{1 / 2}\right\|_{L(\log L)^{n}} \\
& \lesssim\left\|\left(\sum_{I} \frac{\left|\left\langle f, \phi_{I}\right\rangle\right|^{2}}{|I|} \chi_{I}\right)^{1 / 2}\right\|_{L(\log L)^{n+1}} \\
& =\left\|S_{1} f\right\|_{L(\log L)^{n+1}} \lesssim\|f\|_{L(\log L)^{n+2}},
\end{aligned}
$$

and

$$
\begin{aligned}
\|S M f\|_{1, \infty} & =\left\|\left(\sum_{I} M_{2}^{\prime}\left(\frac{\left\langle f, \phi_{I}\right\rangle}{|I|^{1 / 2}} \chi_{I}\right)^{2}\right)^{1 / 2}\right\|_{1, \infty} \\
& \lesssim\left\|\left(\sum_{I} \frac{\left|\left\langle f, \phi_{I}\right\rangle\right|^{2}}{|I|} \chi_{I}\right)^{1 / 2}\right\|_{1}=\left\|S_{1} f\right\|_{1} \lesssim\|f\|_{L \log L} .
\end{aligned}
$$

On the other hand,

$$
\begin{aligned}
M S f(x, y) & =\sup _{I} \frac{1}{|I|^{1 / 2}}\left(\sum_{J} \frac{\left|\left\langle\phi_{R}, f\right\rangle\right|^{2}}{|J|} \chi_{J}(y)\right)^{1 / 2} \chi_{I}(x) \\
& \leq\left(\sum_{J} \frac{\left(\sup _{I} \frac{1}{|I|^{1 / 2}}\left|\left\langle\phi_{R}, f\right\rangle\right| \chi_{I}(x)\right)^{2}}{|J|} \chi_{J}(y)\right)^{1 / 2} .
\end{aligned}
$$


This is essentially $S M$ with the roles of $I$ and $J$ reversed. The same arguments as above can now be applied.

Finally,

$$
\begin{aligned}
S S f(x, y) & =\left(\sum_{R} \frac{\left|\left\langle\phi_{R}, f\right\rangle\right|^{2}}{|R|} \chi_{R}(x, y)\right)^{1 / 2} \\
& =\left[\sum_{I} \sum_{J} \frac{1}{|J|}\left|\left\langle\phi_{J}, \frac{\left\langle f, \phi_{I}\right\rangle}{|I|^{1 / 2}} \chi_{I}(x)\right\rangle\right|^{2} \chi_{J}(y)\right]^{1 / 2} \\
& =\left[\sum_{I} S_{2}\left(\frac{\left\langle f, \phi_{I}\right\rangle}{|I|^{1 / 2}} \chi_{I}\right)(x, y)^{2}\right]^{1 / 2}
\end{aligned}
$$

so that the same proof works.

\section{Bi-parameter multipliers}

Given a vector $\vec{t}=\left(t_{1}, \ldots, t_{2 d}\right) \in \mathbb{R}^{2 d}$, denote $\rho_{1}(\vec{t})=\left(t_{1}, t_{3}, \ldots, t_{2 d-1}\right)$ and $\rho_{2}(\vec{t})=\left(t_{2}, t_{4}, \ldots, t_{2 d}\right)$, which are both vectors in $\mathbb{R}^{d}$. For multi-indices of nonnegative integers $\alpha$, we set $\left|\rho_{1}(\alpha)\right|=\alpha_{1}+\alpha_{3}+\cdots+\alpha_{2 d-1}$, and similarly for $\left|\rho_{2}(\alpha)\right|$. Conversely, for $1 \leq j \leq d$, let $\overrightarrow{t_{j}}=\left(t_{2 j-1}, t_{2 j}\right) \in \mathbb{R}^{2}$, so that $\vec{t}=\left(\overrightarrow{t_{1}}, \ldots, \overrightarrow{t_{d}}\right)$.

Definition. Let $m: \mathbb{R}^{2 d} \rightarrow \mathbb{C}$ be smooth away the origin and uniformly bounded. We say $m$ is a bi-parameter multiplier if

$$
\left|\partial^{\alpha} m(\vec{t})\right| \lesssim\left\|\rho_{1}(\vec{t})\right\|^{-\left|\rho_{1}(\alpha)\right|}\left\|\rho_{2}(\vec{t})\right\|^{-\left|\rho_{2}(\alpha)\right|}
$$

for all vectors $\alpha$ with $|\alpha| \leq 2 d(d+3)$, where $\|\cdot\|$ is the Euclidean norm on $\mathbb{R}^{d}$.

Given such a multiplier $m$ on $\mathbb{R}^{2 d}$ and $L^{1}$ functions $f_{1}, \ldots, f_{d}: \mathbb{T}^{2} \rightarrow \mathbb{C}$, we define the associated multiplier operator $\Lambda_{m}^{(2)}\left(f_{1}, \ldots, f_{d}\right): \mathbb{T}^{2} \rightarrow \mathbb{C}$ as

$$
\Lambda_{m}^{(2)}\left(f_{1}, \ldots, f_{d}\right)(\vec{x})=\sum_{\vec{t} \in \mathbb{Z}^{2 d}} m(\vec{t}) \widehat{f}_{1}\left(\vec{t}_{1}\right) \cdots \widehat{f}_{d}\left(\vec{t}_{d}\right) e^{2 \pi i \vec{x} \cdot\left(\vec{t}_{1}+\cdots+\vec{t}_{d}\right)}
$$

Consider the following theorem.

Theorem 6.1. For any bi-parameter multiplier $m$ on $\mathbb{R}^{2 d}$, it follows that $\Lambda_{m}^{(2)}: L^{p_{1}} \times \cdots \times L^{p_{d}} \rightarrow L^{p}$ for $1<p_{j}<\infty$ and $1 / p_{1}+\cdots+1 / p_{d}=1 / p$. If any or all of the $p_{j}$ are equal to 1 , this still holds with $L^{p}$ replaced by $L^{p, \infty}$ and $L^{p_{j}}$ replaced by $L \log L$. In particular, $\Lambda_{m}^{(2)}: L \log L \times \cdots \times L \log L \rightarrow L^{1 / d, \infty}$. 
We focus only the bi-linear $d=2$ case, but this makes no substantiative difference in the proof. Note that in this case, the bi-parameter multiplier condition can be stated

$$
\left|\partial^{(\alpha, \beta)} m(\vec{s}, \vec{t})\right| \lesssim\left\|\left(s_{1}, t_{1}\right)\right\|^{-\alpha_{1}-\beta_{1}}\left\|\left(s_{2}, t_{2}\right)\right\|^{-\alpha_{2}-\beta_{2}}
$$

for all two-dimensional indices $\alpha, \beta$ with $|\alpha|,|\beta| \leq 10$.

It is by now a well established fact (see $[14,15,17])$ that the study of multiplier operators of various sorts can be reduced to the study of finitely many discrete paraproducts. For $f, g: \mathbb{T}^{2} \rightarrow \mathbb{C}$, the bi-parameter bi-linear paraproducts are defined by

$$
T^{a, b}(f, g)(x, y)=\sum_{R} \frac{1}{|R|^{1 / 2}}\left\langle\phi_{R}^{1}, f\right\rangle\left\langle\phi_{R}^{2}, g\right\rangle \phi_{R}^{3}(x, y),
$$

for $a, b=1,2,3$, where $\phi_{R}^{1}, \phi_{R}^{2}$, and $\phi_{R}^{3}$ are each the tensor product of two normalized adapted families, as in the previous secton. The sum is over all dyadic rectangles $R$. Further, if $\phi_{R}^{i}=\phi_{I}^{i} \otimes \phi_{J}^{i}$, then $\int_{\mathbb{T}} \phi_{I}^{i} d x=0$ for $i \neq a$ and $\int_{\mathbb{T}} \phi_{J}^{i} d x=0$ for $i \neq b$.

In order to establish Theorem 6.1, we need only prove each paraproduct satisfies the same bounds. First, the following lemma is a well-known characterization of weak- $L^{p}$. A proof is given in [1].

Lemma 6.2. Fix $0<p<\infty$ and $f: \mathbb{T}^{d} \rightarrow \mathbb{C}$. Suppose that for every measurable set $|E|>0$ in $\mathbb{T}^{d}$, we can choose a subset $E^{\prime} \subseteq E$ with $\left|E^{\prime}\right|>|E| / 2$ and $\left|\left\langle f, \chi_{E^{\prime}}\right\rangle\right| \leq A|E|^{1-1 / p}$. Then, $\|f\|_{p, \infty} \lesssim A$. Conversely, if $\|f\|_{p, \infty} \leq A$, then for any measurable set $|E|>0$ there exists $E^{\prime} \subseteq E$ with $\left|E^{\prime}\right|>|E| / 2$ and $\left|\left\langle f, \chi_{E^{\prime}}\right\rangle\right| \lesssim A|E|^{1-1 / p}$.

Theorem 6.3. $T^{a, b}: L^{p_{1}} \times L^{p_{2}} \rightarrow L^{p}$ for $1<p_{1}, p_{2}<\infty$ and $1 / p_{1}+1 / p_{2}=1 / p$. If $p_{1}$ or $p_{2}$ or both are equal to 1 , this still holds with $L^{p}$ replaced by $L^{p, \infty}$ and $L^{p_{j}}$ replaced by $L \log L$.

Proof. We will assume $a=1$ and $b=2$, as the other cases will follow similarly.

First, suppose $p>1$. Then, necessarily $p_{1}, p_{2}>1$ and $1<p^{\prime}<\infty$. Note, $1 / p_{1}+1 / p_{2}+1 / p^{\prime}=1$. Fix $h \in L^{p^{\prime}}(\mathbb{T})$ with $\|h\|_{p^{\prime}} \leq 1$. Then,

$$
\begin{aligned}
\left|\left\langle T^{1,2}(f, g), h\right\rangle\right| & \leq \sum_{R} \frac{1}{|R|^{1 / 2}}\left|\left\langle\phi_{R}^{1}, f\right\rangle\right|\left|\left\langle\phi_{R}^{2}, g\right\rangle\right|\left|\left\langle\phi_{R}^{3}, h\right\rangle\right| \\
& =\int_{\mathbb{T}^{2}} \sum_{R} \frac{\left|\left\langle\phi_{R}^{1}, f\right\rangle\right|}{|R|^{1 / 2}} \frac{\left|\left\langle\phi_{R}^{2}, g\right\rangle\right|}{|R|^{1 / 2}} \frac{\left|\left\langle\phi_{R}^{3}, h\right\rangle\right|}{|R|^{1 / 2}} \chi_{R}(x, y) d x d y .
\end{aligned}
$$


Concentrating on the integrand,

$$
\begin{aligned}
\sum_{R} \frac{\left|\left\langle\phi_{R}^{1}, f\right\rangle\right|}{|R|^{1 / 2}} \frac{\left|\left\langle\phi_{R}^{2}, g\right\rangle\right|}{|R|^{1 / 2}} \frac{\left|\left\langle\phi_{R}^{3}, h\right\rangle\right|}{|R|^{1 / 2}} \chi_{R}(x, y)= \\
=\sum_{I} \sum_{J} \frac{\left|\left\langle\phi_{R}^{1}, f\right\rangle\right|}{|R|^{1 / 2}} \frac{\left|\left\langle\phi_{R}^{2}, g\right\rangle\right|}{|R|^{1 / 2}} \frac{\left|\left\langle\phi_{R}^{3}, h\right\rangle\right|}{|R|^{1 / 2}} \chi_{R}(x, y) \\
\leq \sum_{I}\left[\left(\frac{1}{|I|^{1 / 2}} \chi_{I}(x) \sup _{J} \frac{\left|\left\langle\phi_{R}^{2}, g\right\rangle\right|}{|J|^{1 / 2}} \chi_{J}(y)\right)\right. \\
\left.\cdot\left(\sum_{J} \frac{\left|\left\langle\phi_{R}^{1}, f\right\rangle\right|}{|R|^{1 / 2}} \frac{\left|\left\langle\phi_{R}^{3}, h\right\rangle\right|}{|R|^{1 / 2}} \chi_{R}(x, y)\right)\right] .
\end{aligned}
$$

Applying Hölder's inequality, the last term is bounded by

$$
S M(g)(x, y)\left(\sum_{I}\left(\sum_{J} \frac{\left|\left\langle\phi_{R}^{1}, f\right\rangle\right|}{|R|^{1 / 2}} \frac{\left|\left\langle\phi_{R}^{3}, h\right\rangle\right|}{|R|^{1 / 2}} \chi_{R}(x, y)\right)^{2}\right)^{1 / 2} .
$$

Applying Hölder to the inner sum,

$$
\begin{aligned}
& \left(\sum_{I}\left(\sum_{J} \frac{\left|\left\langle\phi_{R}^{1}, f\right\rangle\right|}{|R|^{1 / 2}} \frac{\left|\left\langle\phi_{R}^{3}, h\right\rangle\right|}{|R|^{1 / 2}} \chi_{R}(x, y)\right)^{2}\right)^{1 / 2} \leq \\
& \leq\left(\sum_{I}\left(\sum_{J} \frac{\left|\left\langle\phi_{R}^{1}, f\right\rangle\right|^{2}}{|R|} \chi_{R}(x, y)\right)\left(\sum_{J} \frac{\left|\left\langle\phi_{R}^{3}, h\right\rangle\right|^{2}}{|R|} \chi_{R}(x, y)\right)\right)^{1 / 2} \\
& \leq\left(\sup _{I} \frac{1}{|I|} \chi_{I}(x) \sum_{J} \frac{\left|\left\langle\phi_{R}^{1}, f\right\rangle\right|^{2}}{|J|} \chi_{J}(y)\right)^{1 / 2}\left(\sum_{I} \sum_{J} \frac{\left|\left\langle\phi_{R}^{3}, h\right\rangle\right|^{2}}{|R|} \chi_{R}(x, y)\right)^{1 / 2} \\
& =M S(f)(x, y) S S(h)(x, y) .
\end{aligned}
$$

Hence,

$$
\begin{aligned}
\left|\left\langle T^{1,2}(f, g), h\right\rangle\right| & \leq \int_{\mathbb{T}^{2}} M S f(x, y) S M g(x, y) \operatorname{SSh}(x, y) d x d y \\
& \leq\|M S f\|_{p_{1}}\|S M g\|_{p_{2}}\|S S h\|_{p^{\prime}} \lesssim\|f\|_{p_{1}}\|g\|_{p_{2}} .
\end{aligned}
$$

As $h$ in the unit ball of $L^{p^{\prime}}$ is arbitrary, we have $\left\|T^{1,2}(f, g)\right\|_{p} \lesssim\|f\|_{p_{1}}\|g\|_{p_{2}}$.

Now assume $1 / 2 \leq p \leq 1$. By interpolation, it is sufficient to show $T^{1,2}: L^{p_{1}} \times L^{p_{2}} \rightarrow L^{p, \infty}$ for all $1 \leq p_{1}, p_{2}<\infty$. Fix $\|f\|_{p_{1}}=1$ if $p_{1}>1$ or $\|f\|_{L \log L}=1$ if $p_{1}=1$. Similarly for $g$ and $p_{2}$. Let $E \subseteq \mathbb{T}^{2}$ with $|E|>0$. By Lemma 6.2 , we will be done if we can find $E^{\prime} \subseteq E,\left|E^{\prime}\right|>|E| / 2$ so that $\left|\left\langle T^{1,2}(f, g), \chi_{E^{\prime}}\right\rangle\right| \lesssim 1 \leq|E|^{1-1 / p}$. 
For $\vec{k} \in \mathbb{N}^{2}$ and $R=I \times J$ a dyadic interval, denote $2^{\vec{k}} R=2^{k_{1}} I \times 2^{k_{2}} J$, and $|\vec{k}|=k_{1}+k_{2}$. Use Theorem 4.1 to write

$$
\phi_{R}^{3}=\sum_{\vec{k} \in \mathbb{N}^{2}} 2^{-10|\vec{k}|} \phi_{R}^{3, \vec{k}}
$$

where each $\phi_{R}^{3, \vec{k}}$ is the normalization of the tensor product of two 0-mean adapted families which are uniformally adapted to $I, J$ respectively. Further, $\operatorname{supp}\left(\phi_{R}^{3, \vec{k}}\right) \subseteq 2^{\vec{k}} R$ for $\vec{k}$ small enough, while $\phi_{I}^{3, \vec{k}}$ is identically 0 otherwise. Now

$$
\left\langle T^{1,2}(f, g), \chi_{E^{\prime}}\right\rangle=\sum_{\vec{k} \in \mathbb{N}^{2}} 2^{-10|\vec{k}|} \sum_{R} \frac{1}{|R|^{1 / 2}}\left\langle\phi_{R}^{1}, f\right\rangle\left\langle\phi_{R}^{2}, g\right\rangle\left\langle\phi_{R}^{3, \vec{k}}, \chi_{E^{\prime}}\right\rangle .
$$

Hence, it suffices to show $\left.\left|\sum\right| R\right|^{-1 / 2}\left\langle\phi_{R}^{1}, f\right\rangle\left\langle\phi_{R}^{2}, g\right\rangle\left\langle\phi_{R}^{3, \vec{k}}, \chi_{E^{\prime}}\right\rangle \mid \lesssim 2^{4|\vec{k}|}$, so long as the underlying constants are independent of $\vec{k}$.

Let $S S^{\vec{k}}$ be the double square operator with $\phi_{R}^{3, \vec{k}}$. For each $\vec{k} \in \mathbb{N}^{2}$, define

$$
\begin{aligned}
\Omega_{-3|\vec{k}|} & =\left\{M S f>C 2^{3|\vec{k}|}\right\} \cup\left\{S M g>C 2^{3|\vec{k}|}\right\}, \\
\widetilde{\Omega}_{\vec{k}} & =\left\{M_{S}\left(\chi_{\Omega_{-3 \mid \vec{k}}}\right)>1 / 100\right\}, \\
\widetilde{\Omega}_{\vec{k}} & =\left\{M_{S}\left(\chi_{\widetilde{\Omega}_{\vec{k}}}\right)>2^{-|\vec{k}|-1}\right\} .
\end{aligned}
$$

and

$$
\Omega=\bigcup_{\vec{k} \in \mathbb{N}^{2}} \widetilde{\widetilde{\Omega}}_{\vec{k}}
$$

Observe, $C$ can be chosen independent of $f$ and $g$ so that $|\Omega|<|E| / 2$. Set $E^{\prime}=E-\Omega=E \cap \Omega^{c}$. Then, $E^{\prime} \subseteq E$ and $\left|E^{\prime}\right|>|E| / 2$.

Fix $\vec{k} \in \mathbb{N}^{2}$, and set $Z_{\vec{k}}=\{M S f=0\} \cup\{S M g=0\} \cup\left\{S S^{\vec{k}} \chi_{E^{\prime}}=0\right\}$. Let $\mathcal{D}$ be any finite collection of dyadic rectangles. Consider three subcollections. Set $\mathcal{D}_{1}=\left\{R \in \mathcal{D}: R \cap Z_{\vec{k}} \neq \emptyset\right\}$. For the remaining rectangles, let $\mathcal{D}_{2}=$ $\left\{R \in \mathcal{D}-\mathcal{D}_{1}: R \subseteq \widetilde{\Omega}_{\vec{k}}\right\}$ and $\mathcal{D}_{3}=\left\{R \in \mathcal{D}-\mathcal{D}_{1}: R \cap \widetilde{\Omega}_{\vec{k}}^{c} \neq \emptyset\right\}$.

If $R \in \mathcal{D}_{1}$, then there is some $(x, y) \in R \cap Z_{\vec{k}}$. Namely, $M S f(x, y)=0$, $S M g(x, y)=0$, or $S S^{\vec{k}}\left(\chi_{E^{\prime}}\right)(x, y)=0$. If it is the first, $\left\langle\phi_{R}^{1}, f\right\rangle=0$. If it is the second, then $\left\langle\phi_{R}^{2}, g\right\rangle=0$, and if it is the third, $\left\langle\phi_{R}^{3, \vec{k}}, \chi_{E^{\prime}}\right\rangle=0$. As this holds for all $R \in \mathcal{D}_{1}$, we have

$$
\sum_{R \in \mathcal{D}_{1}} \frac{1}{|R|^{1 / 2}}\left|\left\langle\phi_{R}^{1}, f\right\rangle\right|\left|\left\langle\phi_{R}^{2}, g\right\rangle\right|\left|\left\langle\phi_{R}^{3, \vec{k}}, \chi_{E^{\prime}}\right\rangle\right|=0 .
$$


Now suppose $R \in \mathcal{D}_{2}$, namely $R \subseteq \widetilde{\Omega}_{\vec{k}}$. For some $\vec{k}, \phi_{R}^{3, \vec{k}}$ is identically 0 and $\left\langle\phi_{R}^{3, \vec{k}}, \chi_{E^{\prime}}\right\rangle=0$. For all others, $\phi_{I}^{3, \vec{k}}$ is supported in $2^{\vec{k}} R$. Let $(x, y) \in 2^{\vec{k}} R$, and observe

$$
M_{S}\left(\chi_{\widetilde{\Omega}_{\vec{k}}}\right)(x, y) \geq \frac{1}{\left|2^{\vec{k}} R\right|} \int_{2^{\vec{k}} R} \chi_{\widetilde{\Omega}_{\vec{k}}} d m \geq \frac{1}{2^{|\vec{k}|}} \frac{1}{|R|} \int_{R} \chi_{\widetilde{\Omega}_{\vec{k}}} d m=2^{-|\vec{k}|}>2^{-|\vec{k}|-1} .
$$

That is, $2^{\vec{k}} R \subseteq \widetilde{\widetilde{\Omega}}_{\vec{k}} \subseteq \Omega$, a set disjoint from $E^{\prime}$. Thus, $\left\langle\phi_{R}^{3, \vec{k}}, \chi_{E^{\prime}}\right\rangle=0$. As this holds for all $R \in \mathcal{D}_{2}$, we have

$$
\sum_{R \in \mathcal{D}_{2}} \frac{1}{|R|^{1 / 2}}\left|\left\langle\phi_{R}^{1}, f\right\rangle\right|\left|\left\langle\phi_{R}^{2}, g\right\rangle\right|\left|\left\langle\phi_{R}^{3, \vec{k}}, \chi_{E^{\prime}}\right\rangle\right|=0 .
$$

Finally, we concentrate on $\mathcal{D}_{3}$. Define $\Omega_{-3|\vec{k}|+1}$ and $\Pi_{-3|\vec{k}|+1}$ by

$$
\begin{aligned}
& \Omega_{-3|\vec{k}|+1}=\left\{M S f>C 2^{3|\vec{k}|-1}\right\}, \\
& \Pi_{-3|\vec{k}|+1}=\left\{I \in \mathcal{D}_{3}:\left|I \cap \Omega_{-3|\vec{k}|+1}\right|>|R| / 100\right\} .
\end{aligned}
$$

Inductively, define for all $n>-3|\vec{k}|+1$,

$$
\begin{aligned}
& \Omega_{n}=\left\{M S f>C 2^{-n}\right\}, \\
& \Pi_{n}=\left\{R \in \mathcal{D}_{3}-\bigcup_{j=-3|\vec{k}|+1}^{n-1} \Pi_{j}:\left|R \cap \Omega_{n}\right|>|R| / 100\right\} .
\end{aligned}
$$

As every $R \in \mathcal{D}_{3}$ is not in $\mathcal{D}_{1}$, that is $M S f>0$ on $R$, it is clear that each $R \in \mathcal{D}_{3}$ will be in one of these collections.

Set $\Omega_{-3|\vec{k}|}^{\prime}=\Omega_{-3|\vec{k}|}$ for symmetry. Define $\Omega_{-3|\vec{k}|+1}^{\prime}$ and $\Pi_{-3|\vec{k}|+1}^{\prime}$ by

$$
\begin{aligned}
& \Omega_{-3|\vec{k}|+1}^{\prime}=\left\{S M g>C 2^{3|\vec{k}|-1}\right\}, \\
& \Pi_{-3|\vec{k}|+1}^{\prime}=\left\{R \in \mathcal{D}_{3}:\left|R \cap \Omega_{-3|\vec{k}|+1}^{\prime}\right|>|R| / 100\right\} .
\end{aligned}
$$

Inductively, define for all $n>-3|\vec{k}|+1$,

$$
\begin{aligned}
& \Omega_{n}^{\prime}=\left\{S M g>C 2^{-n}\right\}, \\
& \Pi_{n}^{\prime}=\left\{R \in \mathcal{D}_{3}-\bigcup_{j=-3|\vec{k}|+1}^{n-1} \Pi_{j}^{\prime}:\left|R \cap \Omega_{n}^{\prime}\right|>|R| / 100\right\} .
\end{aligned}
$$

Again, all $R \in \mathcal{D}_{3}$ must be in one of these collections. 
Choose an integer $N$ big enough so that $\Omega_{-N}^{\prime \prime}=\left\{S S^{\vec{k}}\left(\chi_{E^{\prime}}\right)>2^{N}\right\}$ has very small measure. In particular, we take $N$ big enough so that $\left|R \cap \Omega_{-N}^{\prime \prime}\right|<$ $|R| / 100$ for all $R \in \mathcal{D}_{3}$, which is possible since $\mathcal{D}_{3}$ is a finite collection. Define

$$
\begin{aligned}
& \Omega_{-N+1}^{\prime \prime}=\left\{S S^{\vec{k}}\left(\chi_{E^{\prime}}\right)>2^{N-1}\right\}, \\
& \Pi_{-N+1}^{\prime \prime}=\left\{R \in \mathcal{D}_{3}:\left|R \cap \Omega_{-N+1}^{\prime \prime}\right|>|R| / 100\right\},
\end{aligned}
$$

and

$$
\begin{aligned}
& \Omega_{n}^{\prime \prime}=\left\{S S^{\vec{k}}\left(\chi_{E^{\prime}}\right)>2^{-n}\right\}, \\
& \Pi_{n}^{\prime \prime}=\left\{R \in \mathcal{D}_{3}-\bigcup_{j=-N+1}^{n-1} \Pi_{j}^{\prime \prime}:\left|R \cap \Omega_{n}^{\prime \prime}\right|>|R| / 100\right\},
\end{aligned}
$$

Again, all $R \in \mathcal{D}_{3}$ must be in one of these collections.

Consider $R \in \mathcal{D}_{3}$, so that $R \cap \widetilde{\Omega}_{\vec{k}}^{c} \neq \emptyset$. Then, there is some $(x, y) \in$ $R \cap \widetilde{\Omega}_{\vec{k}}^{c}$ which implies $\left|R \cap \Omega_{-3|\vec{k}|}\right| /|R| \leq M_{S}\left(\chi_{\Omega_{-3|\vec{k}|}}\right)(x, y) \leq 1 / 100$. Write $\Pi_{n_{1}, n_{2}, n_{3}}=\Pi_{n_{1}} \cap \Pi_{n_{2}}^{\prime} \cap \Pi_{n_{3}}^{\prime \prime}$. So,

$$
\begin{aligned}
\sum_{R \in \mathcal{D}_{3}} & \frac{1}{|R|^{1 / 2}}\left|\left\langle\phi_{R}^{1}, f\right\rangle\right|\left|\left\langle\phi_{R}^{2}, g\right\rangle\right|\left|\left\langle\phi_{R}^{3, \vec{k}}, \chi_{E^{\prime}}\right\rangle\right| \\
& =\sum_{n_{1}, n_{2}>-3|\vec{k}|, n_{3}>-N}\left[\sum_{R \in \Pi_{n_{1}, n_{2}, n_{3}}} \frac{1}{|R|^{1 / 2}}\left|\left\langle\phi_{R}^{1}, f\right\rangle\right|\left|\left\langle\phi_{R}^{2}, g\right\rangle\right|\left|\left\langle\phi_{R}^{3, \vec{k}}, \chi_{E^{\prime}}\right\rangle\right|\right] \\
& =\sum_{n_{1}, n_{2}>-3|\vec{k}|, n_{3}>-N}\left[\sum_{R \in \Pi_{n_{1}, n_{2}, n_{3}}} \frac{\left|\left\langle\phi_{R}^{1}, f\right\rangle\right|}{|R|^{1 / 2}} \frac{\left|\left\langle\phi_{R}^{2}, g\right\rangle\right|}{|R|^{1 / 2}} \frac{\left|\left\langle\phi_{R}^{3, \vec{k}}, \chi_{E^{\prime}}\right\rangle\right|}{|R|^{1 / 2}}|R|\right]
\end{aligned}
$$

Suppose $R \in \Pi_{n_{1}, n_{2}, n_{3}}$. If $n_{1}>-3|\vec{k}|+1$, then $R \in \Pi_{n_{1}}$, which in particular says $R \notin \Pi_{n_{1}-1}$. So, $\left|R \cap \Omega_{n_{1}-1}\right| \leq|R| / 100$. If $n_{1}=-3|\vec{k}|+1$, then we still have $\left|R \cap \Omega_{-3|\vec{k}|}\right| \leq|R| / 100$, as $R \in \mathcal{D}_{3}$. Similarly, If $n_{2}>-3 k+1$, then $R \in \Pi_{n_{2}}^{\prime}$, which in particular says $R \notin \Pi_{n_{2}-1}^{\prime}$. So, $\left|R \cap \Omega_{n_{2}-1}^{\prime}\right| \leq|R| / 100$. If $n_{2}=-3|\vec{k}|+1$, then we still have $\left|R \cap \Omega_{-3|\vec{k}|}^{\prime}\right|=\left|R \cap \Omega_{-3|\vec{k}|}\right| \leq|R| / 100$, as $R \in \mathcal{D}_{3}$. Finally, if $n_{3}>-N+1$, then $R \notin \Pi_{n_{3}-1}^{\prime \prime}$ and $\left|R \cap \Omega_{n_{3}-1}^{\prime \prime}\right| \leq$ $|R| / 100$. If $n_{3}=-N+1$, then $\left|R \cap \Omega_{-N}^{\prime \prime}\right| \leq|R| / 100$ by the choice of $N$. So, $\left|R \cap \Omega_{n_{1}-1}^{c} \cap \Omega_{n_{2}-1}^{\prime c} \cap \Omega_{n_{3}-1}^{\prime \prime c}\right| \geq \frac{97}{100}|R|$. Let $\Omega_{n_{1}, n_{2}, n_{3}}=\bigcup\left\{R: R \in \Pi_{n_{1}, n_{2}, n_{3}}\right\}$. Then,

$$
\left|R \cap \Omega_{n_{1}-1}^{c} \cap \Omega_{n_{2}-1}^{c c} \cap \Omega_{n_{3}-1}^{\prime c} \cap \Omega_{n_{1}, n_{2}, n_{3}}\right| \geq \frac{97}{100}|R|
$$


for all $R \in \Pi_{n_{1}, n_{2}, n_{3}}$. Further,

$$
\begin{aligned}
& \sum_{R \in \Pi_{n_{1}, n_{2}, n_{3}}} \frac{\left|\left\langle\phi_{R}^{1}, f\right\rangle\right|}{|R|^{1 / 2}} \frac{\mid\left\langle\phi_{R}^{2}, g\right\rangle}{|R|^{1 / 2}} \frac{\left|\left\langle\phi_{R}^{3, \vec{k}}, \chi_{E^{\prime}}\right\rangle\right|}{|R|^{1 / 2}}|R| \\
& \lesssim \sum_{R \in \Pi_{n_{1}, n_{2}, n_{3}}} \frac{\left|\left\langle\phi_{R}^{1}, f\right\rangle\right|}{|R|^{1 / 2}} \frac{\left|\left\langle\phi_{R}^{2}, g\right\rangle\right|}{|R|^{1 / 2}} \frac{\left|\left\langle\phi_{R}^{3, \vec{k}}, \chi_{E^{\prime}}\right\rangle\right|}{|R|^{1 / 2}} \\
& \quad \times\left|R \cap \Omega_{n_{1}-1}^{c} \cap \Omega_{n_{2}-1}^{c} \cap \Omega_{n_{3}-1}^{\prime c} \cap \Omega_{n_{1}, n_{2}, n_{3}}\right| \\
& =\int_{\Omega_{n_{1}-1}^{c} \cap \Omega_{n_{2}-1}^{\prime c} \cap \Omega_{n_{3}-1}^{\prime \prime} \cap \Omega_{n_{1}, n_{2}, n_{3}}} \chi_{R}(x, y) \\
& \quad \sum_{R \in \Pi_{n_{1}, n_{2}, n_{3}}} \frac{\left|\left\langle\phi_{R}^{1}, f\right\rangle\right|}{|R|^{1 / 2}} \frac{\left|\left\langle\phi_{R}^{2}, g\right\rangle\right|}{|R|^{1 / 2}} \frac{\left|\left\langle\phi_{R}^{3, \vec{k}}, \chi_{E^{\prime}}\right\rangle\right|}{|R|^{1 / 2}} d x d y \\
& \leq \int_{\Omega_{n_{1}-1}^{c} \cap \Omega_{n_{2}-1}^{\prime c} \cap \Omega_{n_{3}-1}^{\prime \prime} \cap \Omega_{n_{1}, n_{2}, n_{3}}} M S f(x, y) S M g(x, y) S S^{\vec{k}}\left(\chi_{E^{\prime}}\right)(x, y) d x d y \\
& \lesssim C^{2} 2^{-n_{1}} 2^{-n_{2}} 2^{-n_{3}}\left|\Omega_{n_{1}, n_{2}, n_{3}}\right| .
\end{aligned}
$$

Note,

$$
\begin{aligned}
\left|\Omega_{n_{1}, n_{2}, n_{3}}\right| & \leq\left|\bigcup\left\{R: R \in \Pi_{n_{1}}\right\}\right| \leq\left|\left\{M_{S}\left(\chi_{\Omega_{n_{1}}}\right)>1 / 100\right\}\right| \\
& \lesssim\left|\Omega_{n_{1}}\right|=\left|\left\{M S f>C 2^{-n_{1}}\right\}\right| \lesssim C^{-p_{1}} 2^{p_{1} n_{1}} .
\end{aligned}
$$

Repeating the argument,

$$
\begin{aligned}
& \left|\Omega_{n_{1}, n_{2}, n_{3}}\right| \lesssim\left|\Omega_{n_{2}}^{\prime}\right|=\left|\left\{S M g>C 2^{-n_{2}}\right\}\right| \lesssim C^{-p_{2}} 2^{p_{2} n_{2}}, \quad \text { and } \\
& \left|\Omega_{n_{1}, n_{2}, n_{3}}\right| \lesssim\left|\Omega_{n_{3}}^{\prime \prime}\right|=\left|\left\{S S^{\vec{k}}\left(\chi_{E^{\prime}}\right)>2^{-n_{3}}\right\}\right| \lesssim 2^{\alpha n_{3}}
\end{aligned}
$$

for any $\alpha \geq 1$. Thus, $\left|\Omega_{n_{1}, n_{2}, n_{3}}\right| \lesssim C^{-p_{1}-p_{2}} 2^{\theta_{1} p_{1} n_{1}} 2^{\theta_{2} p_{2} n_{2}} 2^{\theta_{3} \alpha n_{3}}$ for any $\theta_{1}+$ $\theta_{2}+\theta_{3}=1,0 \leq \theta_{1}, \theta_{2}, \theta_{3} \leq 1$. Hence,

$$
\begin{aligned}
\sum_{R \in \mathcal{D}_{3}} \frac{1}{|R|^{1 / 2}}\left|\left\langle\phi_{R}^{1}, f\right\rangle\right|\left|\left\langle\phi_{R}^{2}, g\right\rangle\right|\left|\left\langle\phi_{R}^{3, \vec{k}}, \chi_{E^{\prime}}\right\rangle\right| & \\
& \lesssim \sum_{n_{1}, n_{2}>-3|\vec{k}|, n_{3}>0} 2^{\left(\theta_{1} p_{1}-1\right) n_{1}} 2^{\left(\theta_{2} p_{2}-1\right) n_{2}} 2^{\left(\theta_{3} \alpha-1\right) n_{3}} \\
& +\sum_{n_{1}, n_{2}>-3|\vec{k}|,-N<n_{3} \leq 0} 2^{\left(\theta_{1} p_{1}-1\right) n_{1}} 2^{\left(\theta_{2} p_{2}-1\right) n_{2}} 2^{\left(\theta_{3} \alpha-1\right) n_{3}} \\
=: A+B . &
\end{aligned}
$$


For the first term, take $\theta_{1}=1 /\left(2 p_{1}\right), \theta_{2}=1 /\left(2 p_{2}\right), \theta_{3}=1-1 /(2 p)$, and $\alpha=1$. For the second term, take $\theta_{1}=1 /\left(3 p_{1}\right), \theta_{2}=1 /\left(3 p_{2}\right), \theta_{3}=1-1 /(3 p)>0$, and $\alpha=2 / \theta_{3}$ to see

$$
\begin{aligned}
A & =\sum_{n_{1}, n_{2}>-3|\vec{k}|, n_{3}>0} 2^{-n_{1} / 2} 2^{-n_{2} / 2} 2^{-n_{3} / 2 p} \lesssim 2^{3|\vec{k}|} 2^{1 / 2 p} \leq 2^{3|\vec{k}|+1}, \\
B & =\sum_{n_{1}, n_{2}>-3|\vec{k}|,-N<n_{3} \leq 0} 2^{-2 n_{1} / 3} 2^{-2 n_{2} / 3} 2^{n_{3}} \leq \sum_{n_{1}, n_{2}>-3|\vec{k}|, n_{3} \leq 0} 2^{-2 n_{1} / 3} 2^{-2 n_{2} / 3} 2^{n_{3}} \lesssim 2^{4|\vec{k}|} .
\end{aligned}
$$

Note, there is no dependence on the number $N$, which depends on $\mathcal{D}$, or $C$, which depends on $E$.

Combining the estimates for $\mathcal{D}_{1}, \mathcal{D}_{2}$, and $\mathcal{D}_{3}$, we see

$$
\sum_{R \in \mathcal{D}} \frac{1}{|R|^{1 / 2}}\left|\left\langle\phi_{R}^{1}, f\right\rangle\right|\left|\left\langle\phi_{R}^{2}, g\right\rangle\right|\left|\left\langle\phi_{R}^{3, \vec{k}}, \chi_{E^{\prime}}\right\rangle\right| \lesssim 2^{4|\vec{k}|},
$$

where the constant has no dependence on the collection $\mathcal{D}$. Hence, as $\mathcal{D}$ is arbitrary, we have

$$
\begin{aligned}
\mid \sum_{R} \frac{1}{|R|^{1 / 2}}\left\langle\phi_{R}^{1}, f\right\rangle & \left\langle\phi_{R}^{2}, g\right\rangle\left\langle\phi_{R}^{3, \vec{k}}, \chi_{E^{\prime}}\right\rangle \mid \\
& \leq \sum_{R} \frac{1}{|R|^{1 / 2}}\left|\left\langle\phi_{R}^{1}, f\right\rangle\right|\left|\left\langle\phi_{R}^{2}, g\right\rangle\right|\left|\left\langle\phi_{R}^{3, \vec{k}}, \chi_{E^{\prime}}\right\rangle\right| \lesssim 2^{4|\vec{k}|},
\end{aligned}
$$

which completes the proof.

It should now be clear that proving the above for $(a, b) \neq(1,2)$ follows by permuting the roles of $M M, M S, S M$, and $S S$. For instance, if $(a, b)=$ $(1,1)$, then we consider $M M f, S S g$, and $S S^{\vec{k}} \chi_{E^{\prime}}$.

\section{Multi-parameter multipliers}

Finally, we would like to consider multipliers, and their corresponding operators, which are multi-parameter. That is, $m$ acts as if the product of $s$ standard multipliers.

For a vector $\vec{t} \in \mathbb{R}^{s d}$ and $1 \leq j \leq s$, let $\rho_{j}(\vec{t})=\left(t_{j}, t_{j+s}, \ldots, t_{j+s(d-1)}\right)$ $\in \mathbb{R}^{d}$. Conversely, for $1 \leq j \leq d$, let $\vec{t}_{j}=\left(t_{s(j-1)+1}, \ldots, t_{j s}\right) \in \mathbb{R}^{s}$ so that $\vec{t}=\left(\overrightarrow{t_{1}}, \ldots, \overrightarrow{t_{d}}\right)$.

Let $m: \mathbb{R}^{s d} \rightarrow \mathbb{C}$ be smooth away from the origin and uniformly bounded. We say $m$ is an $s$-parameter multiplier if

$$
\left|\partial^{\alpha} m(\vec{t})\right| \lesssim \prod_{j=1}^{s}\left\|\rho_{j}(\vec{t})\right\|^{-\left|\rho_{j}(\alpha)\right|}
$$

for all indices $|\alpha| \leq s d(d+3)$, where $\|\cdot\|$ is the Euclidean norm on $\mathbb{R}^{d}$. 
Given such a multiplier $m$ on $\mathbb{R}^{s d}$ and $L^{1}$ functions $f_{1}, \ldots, f_{d}: \mathbb{T}^{s} \rightarrow \mathbb{C}$, we define the associated multiplier operator $\Lambda_{m}^{(s)}\left(f_{1}, \ldots, f_{d}\right): \mathbb{T}^{s} \rightarrow \mathbb{C}$ as

$$
\Lambda_{m}^{(s)}\left(f_{1}, \ldots, f_{d}\right)(\vec{x})=\sum_{\vec{t} \in \mathbb{Z}^{s d}} m(\vec{t}) \widehat{f}_{1}\left(\vec{t}_{1}\right) \cdots \widehat{f}_{d}\left(\vec{t}_{d}\right) e^{2 \pi i \vec{x} \cdot\left(\vec{t}_{1}+\cdots+\vec{t}_{d}\right)} .
$$

The familiar $L^{p}$ estimates of still hold with minor modifications.

Theorem 7.1. For any s-parameter multiplier $m$ on $\mathbb{R}^{s d}$, it follows that $\Lambda_{m}^{(s)}: L^{p_{1}} \times \cdots \times L^{p_{d}} \rightarrow L^{p}$ for $1<p_{j}<\infty$ and $1 / p_{1}+\cdots+1 / p_{d}=1 / p$. If any or all of the $p_{j}$ are equal to 1 , this still holds with $L^{p}$ replaced by $L^{p, \infty}$ and $L^{p_{j}}$ replaced by $L(\log L)^{s-1}$. In particular, $\Lambda_{m}^{(s)}: L(\log L)^{s-1} \times \cdots \times L(\log L)^{s-1} \rightarrow$ $L^{1 / d, \infty}$.

In view of these results, we now have a good perception of the heuristics. Away from $p_{j}=1$, each of these operators act the same. However, it is these endpoint cases which are the most interesting. Each time we go up a parameter, we "gain a log" at the endpoint.

Just as in the bi-parameter case, we can reduce to paraproducts. We say $Q \subset \mathbb{T}^{s}$ is a dyadic rectangle if $Q=I_{1} \times \cdots \times I_{s}$ for dyadic intervals $I_{j}$. Let $\varphi_{Q}: \mathbb{T}^{s} \rightarrow \mathbb{C}$ be the $s$-fold tensor product of adapted families. The appropriate (bi-linear) paraproducts in this setting are

$$
T_{\epsilon}^{a_{1}, \ldots, a_{s}}(f, g)(\vec{x})=\sum_{Q} \frac{1}{|Q|^{1 / 2}}\left\langle\phi_{Q}^{1}, f\right\rangle\left\langle\phi_{Q}^{2}, g\right\rangle \phi_{Q}^{3}(\vec{x})
$$

where the sum is over all dyadic rectangles $Q$. Each $a_{j}$ ranges over 1,2,3. If $\phi_{Q}^{i}=\phi_{I_{1}}^{i} \otimes \cdots \otimes \phi_{I_{s}}^{i}$, then $\int_{\mathbb{T}} \phi_{I_{j}}^{i} d x=0$ whenever $i \neq a_{j}$.

To complete the proof on $s$-parameter multiplier operators, it suffices to show the associated paraproducts satisfy the same bounds. The same stopping time argument works equally well in all dimensions, given the correct $s$-fold hybrid operators. Therefore, we will understand the paraproducts if we can show each $s$-fold hybrid operator maps $L^{p} \rightarrow L^{p}$ for $1<p<\infty$ and $L(\log L)^{s-1} \rightarrow L^{1, \infty}$.

For illustrative purposes, we show this for one specific operator when $s=3$. For $f: \mathbb{T}^{3} \rightarrow \mathbb{C}$ define

$$
\operatorname{SSMf}(x, y, z)=\left(\sum_{I_{1}} \sum_{I_{2}} \frac{\left(\sup _{I_{3}} \frac{1}{\left|I_{3}\right|^{1 / 2}}\left|\left\langle\phi_{Q}, f\right\rangle\right| \chi_{I_{3}}(z)\right)^{2}}{\left|I_{1}\right|\left|I_{2}\right|} \chi_{I_{1}}(x) \chi_{I_{2}}(y)\right)^{1 / 2} .
$$

Using the same notational conveniences as before,

$$
S S M f=\left(\sum_{I_{1}} \sum_{I_{2}} M_{3}^{\prime}\left(\frac{\left\langle f, \phi_{I_{1}} \otimes \phi_{I_{2}}\right\rangle}{\left|I_{1}\right|^{1 / 2}\left|I_{2}\right|^{1 / 2}} \chi_{I_{1}} \chi_{I_{2}}\right)^{2}\right)^{1 / 2} .
$$


So,

$$
\begin{aligned}
\|S S M f\|_{p} & =\left\|\left(\sum_{I_{1}} \sum_{I_{2}} M_{3}^{\prime}\left(\frac{\left\langle f, \phi_{I_{1}} \otimes \phi_{I_{2}}\right\rangle}{\left|I_{1}\right|^{1 / 2}\left|I_{2}\right|^{1 / 2}} \chi_{I_{1}} \chi_{I_{2}}\right)^{2}\right)^{1 / 2}\right\|_{p} \\
& \lesssim\left\|\left(\sum_{I_{1}} \sum_{I_{2}} \frac{\left|\left\langle f, \phi_{I_{1}} \otimes \phi_{I_{2}}\right\rangle\right|^{2}}{\left|I_{1}\right|\left|I_{2}\right|} \chi_{I_{1}} \chi_{I_{2}}\right)^{1 / 2}\right\|_{p} \\
& =\left\|\left(\sum_{I_{1}} S_{2}\left(\frac{\left\langle f, \phi_{I_{1}}\right\rangle}{\left|I_{1}\right|^{1 / 2}} \chi_{I_{1}}\right)^{2}\right)^{1 / 2}\right\|_{p} \lesssim\left\|\left(\sum_{I_{1}} \frac{\left|\left\langle f, \phi_{I_{1}}\right\rangle\right|^{2}}{\left|I_{1}\right|} \chi_{I_{1}}\right)^{1 / 2}\right\|_{p} \\
& =\left\|S_{1} f\right\|_{p} \lesssim\|f\|_{p},
\end{aligned}
$$

and

$$
\begin{aligned}
\|S S M f\|_{1, \infty} & =\left\|\left(\sum_{I_{1}} \sum_{I_{2}} M_{3}^{\prime}\left(\frac{\left\langle f, \phi_{I_{1}} \otimes \phi_{I_{2}}\right\rangle}{\left|I_{1}\right|^{1 / 2}\left|I_{2}\right|^{1 / 2}} \chi_{I_{1}} \chi_{I_{2}}\right)^{2}\right)^{1 / 2}\right\|_{1, \infty} \\
& \lesssim\left\|\left(\sum_{I_{1}} S_{2}\left(\frac{\left\langle f, \phi_{I_{1}}\right\rangle}{\left|I_{1}\right|^{1 / 2}} \chi_{I_{1}}\right)^{2}\right)^{1 / 2}\right\|_{1} \lesssim\left\|S_{1} f\right\|_{L \log L} \lesssim\|f\|_{L(\log L)^{2}}
\end{aligned}
$$

The recipe for arbitrary $s$-fold hybrid operators should now be clear. Each such operator is pointwise smaller than one of the form $S S \ldots S M M \ldots M$. In this case, the $M \ldots M M$ part is bounded by $M_{j} \circ M_{j+1} \circ \cdots \circ M_{s}$. Repeated iterations of Fefferman-Stein eliminate these $M_{j}$, while the remaining $S S \ldots S$ part can be dealt with as usual.

Acknowledgements. The author would like to extend his sincere gratitude to Camil Muscalu. The author received support from both NSF and Department of Defense Graduate Fellowships.

\section{References}

[1] Auscher, P., Hoffman, S., Muscalu, C., Tao, T. and Thiele, C.: Carleson measures, trees, extrapolation, and $T(b)$ theorems. Publ. Mat. 46 (2002), 257-325.

[2] Bennett, C. and Sharpley, R.: Weak-type inequalities for $H^{p}$ and BMO. In Harmonic analysis in Euclidean spaces (Proc. Sympos. Pure Math., Williams Coll., Williamstown, Mass. (1978), Part 1, 201-229. Proc. Sympos. Pure Math.35. Amer. Math. Soc., Providence, R.I., 1979.

[3] Bennett, C. and Sharpley, R.: Interpolation of operators. Pure and Applied Mathematics 129. Academic Press, Boston, MA, 1988.

[4] Christ, M. And Journé, J.-L.: Polynomial growth estimates for multilinear singular integral operators. Acta Math. 159 (1987), 51-80. 
[5] Coifman, R. and Meyer, Y.: Ondelettes et Opérateurs, III: Opérateurs multilinéaires. Actualités Mathématiques. Hermann, Paris, 1991.

[6] Fefferman, C.: Estimates for double Hilbert transforms. Studia Math. 44 (1972), 1-15.

[7] Fefferman, C. and Stein, E. M.: Some maximal inequalities. Amer. J. Math. 93 (1971), 107-15.

[8] García-Cuerva, J. and Rubio de Francia, J. L.: Weighted Norm Inequalities and Related Topics. North-Holland Mathematics Studies 116. North-Holland, Amsterdam, 1985.

[9] Grafakos, L. and Torres, R.: Multilinear Calderón-Zygmund theory. Adv. Math. 165 (2002), 124-164.

[10] Journé, J.-L.: Calderón-Zygmund operators on product spaces. Rev. Mat. Iberoamericana 1 (1985), no. 3, 55-91.

[11] Kenig, C. and Stein, E. M.: Multilinear estimates and fractional integration. Math. Res. Lett. 6 (1999), 1-15.

[12] Marcinkiewicz, J.: Sur les multiplicateurs des séries de Fourier. Studia Math. 8 (1939), 78-91.

[13] Muscalu, C., Pipher, J., Tao, T. and Thiele, C.: Bi-parameter paraproducts. Acta Math. 193 (2004), 269-296.

[14] Muscalu, C., Pipher, J., TaO, T. and Thiele, C.: Multi-parameter paraproducts. Rev. Mat. Iberoam. 22 (2006), no. 3, 963-976.

[15] Muscalu, C., TaO, T. and Thiele, C.: Multi-linear operators given by singular multipliers. J. Amer. Math. Soc. 15 (2002), 469-496.

[16] Stein, E. M.: Note on the class L $\log L$. Studia Math. 32 (1969), 305-310.

[17] Workman, J. T.: End-point estimates and multi-parameter paraproducts on higher dimensional tori. Available at arXiv.org/abs/0806 .0197, 2008.

Recibido: 11 de septiembre de 2008

Revisado: 22 de noviembre de 2008

John T. Workman

5811 Sable Dr.

Alexandria, VA 22303

United States

johntylerworkman@gmail.com 